\title{
Clase social y estrategias parentales de apoyo a los estudiantes en pandemia. Resultados para Chile del International COVID-19 Impact on Parental Engagement Study *
}

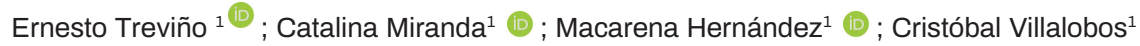 \\ ${ }^{1}$ Pontificia Universidad Católica de Chile (UC)
}

Resumen. La irrupción de la pandemia (COVID-19) y el tránsito a la educación remota posiciona a las familias en el proceso y experiencia educativa de sus hijos e hijas como uno de especial importancia (Bonal y González, 2020). El objetivo del artículo es explorar las prácticas educativas de la clase media-profesional chilena en período de crisis sanitaria. Para ello se utiliza la base de datos del estudio transnacional, International COVID-19 Impact on Parental Engagement Study (ICIPES) que agrupó a 23 países. En el caso chileno se recogieron 1.597 respuestas que permitieron describir las prácticas educativas ejecutadas por los grupos sociales, además, de testear la relación entre ambas variables. Se concluye que existe un involucramiento parental transversal en el proceso y experiencia de aprendizaje durante la pandemia. Sin embargo, hay diferencias en las prácticas educativas. Mientras los padres y madres no profesionales buscan prácticas de fortalecimiento educativo, aquellos que componen el grupo profesional priorizan la participación del menor en actividades extra-educativas que estimulen su aprendizaje cognitivo. Además, algunas prácticas educativas formales y no formales están asociadas con la clase social de las familias y, por ende, reafirma la desigualdad educativa que se reproduce según los recursos económicos que tiene una persona.

Palabras clave: pandemia; prácticas educativas; familia; clase social.

Classe social e estratégias parentais de apoio aos estudantes na pandemia. Resultados para o Chile do International COVID-19 Impact on Parental Engagement Study

Resumo. A irrupção da pandemia COVID-19 e a transição para a educação remota situam as famílias no processo e na experiência educacional de seus filhos e filhas numa posição de especial importância (Bonal e González, 2020). O objetivo do artigo é explorar as práticas educacionais da classe média profissional chilena num período de crise sanitária. Para isso, utiliza-se o banco de dados do estudo transnacional, International COVID-19 Impact on Parental Engagement Study (ICIPES), que agrupou 23 países. No caso chileno, coletaram-se 1.597 respostas para descrever as práticas educacionais realizadas pelos grupos sociais, além de testar a relação entre as duas variáveis. Conclui-se que há um envolvimento parental transversal no processo e experiência de aprendizagem durante a pandemia. No entanto, há diferenças nas práticas educacionais. Enquanto pais e mães não profissionais procuram práticas de fortalecimento da educação, aqueles que fazem parte do grupo profissional priorizam a participação da criança em atividades extraescolares que estimulam sua aprendizagem cognitiva. Além disso, algumas práticas educacionais formais e não formais estão associadas à classe social das famílias e, portanto, reafirmam a desigualdade educacional que se reproduz de acordo com os recursos econômicos que uma pessoa possui.

Palavras-chave: pandemia; práticas educacionais; familia; classe social.

Social class and parental support strategies for students in pandemic: Results for Chile from the International COVID-19 Impact on Parental Engagement Study.

Abstract. The irruption of the pandemic (COVID-19) and the transition to remote education positions families in the educational process and experience of their children as one of special importance (Bonal \& González, 2020). The aim of the article is to explore the educational practices of the Chilean middle-professional class in a period of health crisis. For this purpose, we used the database of the transnational study, International COVID-19 Impact on Parental Engagement Study (ICIPES), which included 23 countries. In the Chilean case, 1,597 responses were collected to describe the educational practices carried out by the social groups, as well as to test the relationship between the two variables. It is concluded that there is a transversal parental involvement in the learning process and experience during the pandemic. However, there are differences in educational practices. While non-professional parents seek educational reinforcement practices, those in the professional group prioritise the child's participation in extra-educational activities that stimulate cognitive learning. Moreover, some formal and non-formal educational practices are associated with the social class of the families and thus reaffirm the educational inequality that is reproduced according to the economic resources a person has.

Keywords: pandemic; educational practices; family; social class

*El proyecto es financiado por la Agencia Nacional de Investigación y Desarrollo (ANID) de Chile. 


\section{Introducción}

La crisis sanitaria experimentada desde el año 2020 hasta la actualidad por la irrupción de la pandemia de la COVID-19 ha provocado la suspensión de las clases presenciales en las instituciones educativas de más de 190 países. A mediados de mayo de 2020, 1.2 billones de estudiantes estaban cursando su año escolar desde sus hogares, de los cuales 160 millones correspondían a estudiantes de América Latina y el Caribe (CEPAL y UNESCO, 2020). En enero de 2021, la región se situaba como aquella con el cierre promedio más prolongado de los centros educativos del mundo (UNESCO, 2021).

Para responder a este escenario, los sistemas educativos deAmérica Latina han implementado distintas iniciativas para favorecer la continuidad educativa, incluyendo modalidades de enseñanza en línea y fuera de línea, la transmisión de programas por televisión y radio, la puesta a disposición de plataformas y portales web con recursos pedagógicos, la entrega de dispositivos tecnológicos y - en menor medida - la ejecución de clases en línea (CEPAL y UNESCO, 2020). Distintos reportes internacionales han posicionado a América Latina y el Caribe como una región especialmente afectada por la pandemia y sus repercusiones por la presencia de profundas desigualdades sociales (Busso y Messina, 2020). Alineado a ello, en el ámbito educativo, el tránsito a la educación remota de emergencia ha sido caracterizado como uno especialmente complejo por las inequidades entre los hogares pertenecientes a distintos grupos sociales, relevando principalmente las brechas tecnológicas preexistentes (OECD, 2020).

En el caso de Chile, el gobierno decretó la suspensión de las clases presenciales a nivel nacional el 16 de marzo de 2020. Al finalizar el año escolar 2020, sólo 1 de cada 10 instituciones educativas habían retomado las clases presenciales (Ministerio de Educación, 2020). A lo largo de este periodo, el foco central de la estrategia ministerial ha consistido en el despliegue de iniciativas principalmente focalizadas en apoyar el tránsito a la educación en línea de los estudiantes, por medio de la puesta a disposición de plataformas online, la entrega de recursos pedagógicos digitales y de capacitaciones a los docentes para las clases virtuales, lo que ha aumentado las brechas educativas y ha deteriorado la capacidad de ejercer el derecho a la educación (Gelber, Castillo, Alarcón, Treviño y Escribano, 2021).

Hasta la fecha, la investigación que ha retratado el tránsito a la educación a distancia desde el lado de los hogares ha evidenciado las condiciones desiguales para el aprendizaje remoto en casa, dando cuenta de las características desiguales de las viviendas, las inequidades respecto de presencia de espacios adecuados para estudiar, las diferencias de disposición de equipos tecnológicos de uso exclusivo para los estudiantes y la calidad de la conexión a internet, entre otros aspectos (Bonal y González, 2020; Ponce, Bellei y Vielma, 2020), todos hallazgos que han alertado acerca de la potencial exacerbación de las inequidades educativas en la era postpandémica. Pese a la valiosa información provista por estos estudios, menor atención se ha prestado - salvo excepciones (como el caso de Bonal y González (2020) para Cataluña - a las prácticas educativas formales e informales desplegadas por las familias en el hogar, que han sido por varias décadas relevadas por la sociología de la educación como una fuente clave de la producción y transmisión de ventajas de la clase media-alta a sus hijos o hijas (e.g., Nogueira, 2010). 
Considerando estos antecedentes, y buscando contribuir en esta última dirección, este artículo utiliza una fuente de datos de un estudio transnacional de 23 países, para focalizarse en el caso de Chile y explorar las prácticas de la clase media profesional y la clase baja no profesional respecto de la experiencia educativa de sus hijos o hijas durante el tránsito a la educación remota. De esta forma, se busca reflexionar en torno al impacto que los desiguales capitales de clase podrían tener en las dinámicas de aprendizaje en los hogares, propiciadas por el cierre de las instituciones escolares y su proyección en la intensificación de las brechas educativas a futuro.

El artículo se divide en cuatro secciones. El primer apartado exhibe el marco teórico y la literatura que enmarca el objetivo de investigación, mientras que el segundo expone los antecedentes principales del sistema escolar chileno y la respuesta nacional ante la irrupción de la pandemia. La tercera sección desarrolla el método del estudio, luego de lo cual se presentan los principales hallazgos. Por último, la sección final sintetiza y discute las conclusiones del estudio.

\section{Clases medias-altas, estrategias parentales y la produccion de ventajas educativas}

El involucramiento parental en la educación de sus hijos e hijas ha sido relevado como un factor clave en el logro de los resultados académicos (Wilder, 2014). Si bien existe una alta variedad de acepciones de este constructo, el involucramiento de las familias puede definirse como la participación de los padres y madres en el proceso y experiencia educativa de sus hijos o hijas (Wilder, 2014). Asumiendo la amplitud del concepto, distintos investigadores han buscado operacionalizar el término, aprehendiéndolo como un continuo entre el involucramiento en/con la escuela, el involucramiento en la escolaridad y el involucramiento con el aprendizaje de los niños, niñas y adolescentes (Goodall y Montgomery, 2014) o bien distinguiendo las esferas del involucramiento en que se ha distinguido entre las prácticas en la escuela y en el hogar (Gubbins y Otero, 2020). Por otra parte, y centrándose en la esfera del hogar, distintos autores han establecido una diferenciación entre las prácticas educativas formales, aquellas dirigidas directamente a apoyar la enseñanza escolar y las prácticas educativas informales, aquellas experiencias en que los estudiantes son expuestos indirecta o espontáneamente a la adquisición de determinados aprendizajes (Skwarchuk, Sowinski y LeFevre, 2014).

Si bien el involucramiento de las familias en la educación de sus hijos o hijas ha sido mencionado como una condición clave para el aprendizaje, por varias décadas la investigación de la sociología de la educación ha alertado que la clase social provee a las familias con variados recursos (capitales) y disposiciones que afectan desigualmente sus posibilidades de participar en el proceso y experiencia educativa de sus hijos e hijas (Bourdieu y Passeron, 1964; Lareau, 2000, 2011; Vincent, 2001). En los últimos años, la literatura en este tema ha transitado desde un foco en los grupos desaventajados hacia los privilegiados, volcándose crecientemente el estudio a la relación de las clases medias con la educación (Reay et al., 2011). Uno de los principales consensos en la investigación al respecto es el posicionamiento de la educación como un espacio clave para la transmisión de ventajas a sus hijos o hijas y la reproducción social de este grupo (Nogueira, 2010). Así, basándose en la teoría de Bourdieu y reconociendo 
que la posesión de capitales de clase no se traduce automáticamente en ventajas, sino que éstos deben ser activados (Lareau, 2001, 2011), la investigación alrededor del mundo ha dado cuenta consistentemente de las prácticas educativas específicas y sofisticadas de las familias de clases medias, destacando su aporte en la obtención y transmisión de ventajas para sus hijos o hijas (Nogueira, 2010). En esta dirección, Nogueira (2010), a partir de una síntesis de la literatura al respecto, sistematiza entre otra - prácticas parentales orientadas al intenso monitoreo de las actividades escolares y a la estimulación temprana del desarrollo cognitivo de sus hijos o hijas en actividades fuera de la escuela y en el hogar como parte del repertorio de acciones realizadas por las familias para activar sus privilegios.

Los trabajos seminales de Lareau al respecto, junto con mapear en sus primeros estudios el papel de la clase social en el involucramiento parental en las escuelas (Lareau, 2000), han avanzado a levantar las diferencias de clase en las prácticas y dinámicas en el hogar (Lareau, 2011). Aun cuando su trabajo etnográfico releva que subsiste un conjunto de experiencias que no varían según la clase social - como los rituales familiares o los episodios de conexión emocional con sus hijos o hijas -, la investigación enfatiza el despliegue de parte de las familias de las clases medias profesionales de lo que denomina la lógica del 'cultivo concertado' (concerted cultivation), marcada por la intensa ejecución de prácticas - favorecidas por sus capitales de clase - dirigidas a la estimulación de los aprendizajes cognitivos y de las habilidades sociales en sus hijos o hijas, en que destaca el énfasis en la organización estructurada de la rutina de los hijos/hijas en el hogar, la promoción de su participación en múltiples actividades extra-educativas (e.g., clases de arte, música, deportes, clubes) y el uso de conversaciones reflexivas y con un lenguaje rico y complejo (Lareau, 2011).

La irrupción de la pandemia de la COVID-19 y el tránsito a la educación remota de emergencia en el hogar posiciona al papel de las familias en el proceso y experiencia educativa de sus hijos o hijas como uno de especial importancia (Bonal y González, 2020). Pese a la novedad de este tránsito, el impacto de la pandemia en la educación ha sido un terreno fértil de investigación. A la fecha, el grueso de los estudios disponibles acerca de la educación en el hogar y centrados en las familias, se han focalizado en dos grandes líneas. Por un lado, un conjunto de trabajos en distintos países del mundo ha estudiado la experiencia de los padres y madres ante la educación en casa. Estas investigaciones han remarcado de manera consistente las dificultades del proceso de enseñanza en el hogar, dando cuenta de situaciones de estrés y agotamiento de parte de las familias en el marco del desafío de balancear sus nuevos roles educativos con las responsabilidades laborales, así como también de los retos que éstas enfrentan por la falta de habilidades pedagógicas y digitales para apoyar a sus hijos o hijas en la enseñanza remota (e.g., Bhamani et al., 2020; Bubb y Jones, 2020; Fontenelle-Tereshchuk, 2021; Parczewska, 2020; Pozas, Letzel y Schneider, 2021). Por otro lado, un segundo grupo de reportes internacionales e investigaciones empíricas se ha enfocado en las condiciones estructurales presentes en los hogares para dar respuesta a las necesidades de la educación remota, relevando las brechas entre clases sociales en aspectos como el tamaño de los hogares, los dispositivos tecnológicos disponibles y la conectividad a internet (e.g., Bonal y González, 2020; OECD, 2020). 
Sin desconocer la importante información provista por este último set de investigaciones y su aporte en develar las inequidades al respecto, actualmente son escasos los estudios sobre las prácticas específicas que han desplegado las familias en la educación en el hogar y el rol de sus capitales de clase en este plano. Una relevante excepción es el trabajo investigativo de Bonal y González (2020) en Cataluña que - a partir de los datos de una encuesta a padres y madres - han dado cuenta de un rol marcadamente distinto entre clases sociales en la enseñanza en el hogar, develando que las familias con más recursos adoptan en mayor medida prácticas informales de educación en el hogar alineadas a la lógica escolar así como también actividades extra-curriculares, y muestran una mayor confianza en sus propias habilidades para responder a las necesidades de aprendizaje de sus hijos o hijas. Buscando contribuir a esta última línea de estudios, este artículo - usando el caso de Chile - explora las prácticas de las familias en la educación de sus hijos/hijas en el hogar, bajo el contexto de la pandemia, poniendo especial énfasis en la clase media profesional y su proyección en la intensificación de las desigualdades educativas a futuro. De esta forma, se busca explorar en las estrategias familiares en un país con un sistema altamente segregado y organizado en torno al mercado (Villalobos y Quaresma, 2015), indagando en la relación entre estrategias familiares y capitales sociales en contextos pandémicos.

\section{El sistema escolar chileno y la respuesta ante la pandemia de la COVID-19}

El sistema escolar chileno ha sido considerado por varias décadas como un caso pionero y radical en la instalación de un modelo de mercado en educación, que se inicia en la década de 1980 en la dictadura militar y ha sido sostenido por los gobiernos democráticos posteriores. En breve, las principales medidas incorporadas en el llamado experimento neoliberal consistieron en la descentralización de la gestión de las escuelas públicas a los municipios, la implementación universal de un subsidio a la demanda por estudiante como mecanismo de financiamiento competitivo de las escuelas y la entrega de incentivos para la expansión de proveedores privados (Bellei, 2015). Estas disposiciones se acompañaron de un sistema escasamente regulado de admisión de los estudiantes a las instituciones escolares y, más adelante, de la introducción de un financiamiento compartido para las familias de escuelas particulares subvencionadas y municipales de enseñanza media ${ }^{1}$. Con posterioridad, este esquema ha sido complementado con un amplio conjunto de políticas de rendición de cuentas con consecuencias, en que destaca la aplicación extensa e intensa de evaluaciones estandarizadas en distintos grados y niveles educativos (SIMCE) y la configuración de una nueva institucionalidad encargada de fiscalizar, evaluar, inspeccionar y sancionar a las escuelas públicas y privadas (Parcerisa y Falabella, 2017).

Si bien Chile ha realizado importantes progresos en las últimas décadas en cobertura y retención, y ha mostrado avances progresivos en los resultados de los estudiantes en pruebas internacionales que lo sitúan entre los mejores países de la región (OECD, 2019a; Santiago et al., 2017), hasta la fecha el sistema educativo chileno persiste como uno de más segregados de la OECD y de América Latina (OECD,

\footnotetext{
1 Al año 2019, el sistema escolar contaba con 2.949.949 estudiantes en educación primaria y secundaria regular y especial, de los cuales un $37 \%$ se educaba en un establecimiento municipal o público, $52 \%$ en uno particular subvencionado, $9 \%$ en uno particular pagado y $2 \%$ de administración delegada (Ministerio de Educación Chile, 2020).
} 
2019b; UNESCO, 2020), y se mantienen profundas inequidades en el rendimiento de los estudiantes según su origen socioeconómico, los cuales reflejan la desigualdad social prevaleciente en el país. En este contexto, entre el año 2014 al 2018 el país se embarcó en un conjunto de reformas dirigidas a mejorar la calidad y equidad educativa, incluyendo iniciativas de largo aliento dirigidas a fortalecer la educación pública, a potenciar la inclusión escolar y a la creación de un nuevo sistema de desarrollo profesional docente (Treviño, 2018).

En el contexto institucional antes descrito, y frente a la irrupción de la pandemia de la COVID-19, Chile decretó el cierre de las instituciones educativas a nivel nacional el 16 de marzo del 2020, situación que se mantuvo hasta julio, momento en el cual se iniciaron los esfuerzos por avanzar a la apertura parcial. Según el catastro del Ministerio de Educación, a diciembre 2020 sólo el 14\% de los centros educativos reanudaron las clases presenciales en el segundo semestre de dicho año (Ministerio de Educación Chile, 2021a). De manera de apoyar la continuidad del proceso educativo, el Ministerio de Educación puso en marcha distintas iniciativas, que incluyeron la implementación del portal web Aprendo en línea, en la cual estudiantes y docentes cuentan con textos escolares, guías didácticas y cuadernos de actividades para todas las asignaturas, organizados por niveles y grados, la viabilidad del acceso a plataformas de educación remota como Google Suite para las instituciones educativas, una serie de programas de formación y capacitaciones a los profesores, y otras medidas dirigidas a incrementar el acceso a dispositivos y conexión a internet para estudiantes focalizados (Gelber et al., 2021).

Los distintos estudios empíricos desarrollados a lo largo del año 2020 en el marco de la educación remota han aportado evidencia de la implementación de la continuidad educativa en el país, dentro de los cuales destacan tres grandes resultados. En primer lugar, la modalidad de educación remota seguida de parte de las instituciones educativas releva importantes brechas según la dependencia administrativa de las escuelas, identificándose una significativa mayor frecuencia del uso de clases virtuales en el sector privado (Elige Educar, 2020a,b). Así, por ejemplo, según una encuesta efectuada en agosto 2020 a una muestra de profesores, mientras el uso de clases en vivo con posibilidad de interacción era efectuado por $85 \%$ de los docentes participantes del sector particular pagado participantes, la cifra disminuía al $52 \%$ entre los profesores de establecimientos particular subvencionado y a sólo 33\% entre aquellos del ámbito público-municipal (Elige Educar, 2020b).

En segundo lugar, los estudios han levantado los importantes desafíos en las experiencias de los profesores en la implementación de la educación remota, que incluyen problemáticas vinculadas a calidad de su conexión a internet, a aspectos técnicos de la conectividad de sus estudiantes, a la falta de competencias digitales, y a dificultades psicoemocionales como la alta sensación de estrés entre ellos (Centro de Investigación Avanzada en Educación, Centro de Investigación para la Educación Inclusiva y EduGlobal, 2020; Educación 2020). 
Por último, desde el lado de los hogares, algunos estudios han develado las profundas brechas en las condiciones para la educación a distancia, mostrando severas diferencias según el nivel social en las características de las viviendas (e.g., tamaño, disposición de un lugar para estudiar, y espacio exterior), la disposición de recursos educativos (e.g., libros), y de dispositivos tecnológicos y conectividad a internet, identificándose una mayor frecuencia de la tenencia de computadores para uso exclusivo del trabajo escolar y una mejor calidad de conexión a internet entre quienes asisten a la educación privada y pertenecen a los grupos socioeconómicamente favorecidos (Educación 2020, 2020; Ponce, Bellei y Vielma, 2020).

Considerando estos hallazgos, la investigación acumulada - ratificando las brechas sociales agudas presentes en el país y el sistema educativo segregado previo a la pandemia - ha ilustrado las profundas inequidades en el tipo de experiencia educativa de los niños, niñas y adolescentes durante el tránsito a la educación remota provocadas tanto por la respuesta de las instituciones educativas como de las condiciones existentes en los hogares. Pese a estos importantes resultados, menor profundización ha existido en torno a las prácticas parentales de educación en casa entre diferentes grupos sociales que permitan visibilizar la producción activa de la inequidad en la educación remota en el hogar, y su proyección a futuro, foco central de este trabajo.

\section{Método}

\subsection{Datos}

A partir de la crisis sanitaria que impactó al sector educativo a nivel global y con ello, en el aprendizaje de los estudiantes, se aplicó el International COVID-19 Impact on Parental Engagement Study (ICIPES). Este estudio es el resultado de una colaboración de 20 instituciones de investigación a nivel mundial, liderado por la Universidad de Bath (Reino Unido). En Chile, la entidad a cargo de la recolección de datos fue la Facultad de Educación de la Pontificia Universidad Católica de Chile. Durante 3 meses se difundió de manera online un cuestionario que buscaba conocer la forma en que madres, padres y cuidadores construían capacidades para comprometerse con el aprendizaje de sus hijos (Osorio-Saez et al., 2021).

En el caso chileno, en total se recogieron 1.597 respuestas, de las cuales el $92 \%$ son madres, padres y apoderados de zona urbana, además el $81 \%$ son mujeres y 19\% hombres. En relación con la composición del grupo familiar, el $64 \%$ plantea vivir con la madre o padre del menor; el $18 \%$ no tiene un compañero permanente; un $8 \%$ vive con una pareja que no es el padre o madre del menor y $11 \%$ prefiere no entregar tal información. Con respecto al menor de edad, el 50\% son mujeres y $49 \%$ son hombres. La distribución de su edad es heterogénea, entre los 6 a 16 años. En general, los menores tienen 1 hermano (42\%), el $31 \%$ no tiene hermanos y $20 \%$ tiene dos; solo el $2 \%$ tiene cuatro hermanos y menos del $1 \%$ tiene cinco o más hermanos.

\subsection{Variables}

La encuesta incluye, a modo de contextualización, preguntas sobre las características del padre, madre o apoderado y sobre el menor. La primera sección de la encuesta indaga en la relación con a las condiciones de aprendizaje en el hogar (conexión a internet, dispositivos tecnológicos y estructuración del aprendizaje, tal 
como el tiempo y preocupación para estudiar), mientras que la segunda sección que engloba las prácticas educativas formales (vías para fortalecer el proceso de aprendizaje y estructuración de las experiencias educativas) y prácticas educativas no formales (actividades espontáneas o cotidianas). En la tabla 1 se exponen las variables que serán consideradas en el análisis.

Tabla 1. Dimensiones de prácticas educativas formales y no formales

\begin{tabular}{|c|c|}
\hline Dimensión & Variables \\
\hline \multirow[t]{6}{*}{$\begin{array}{l}\text { Prácticas de for- } \\
\text { talecimiento edu- } \\
\text { cativo }\end{array}$} & $\begin{array}{l}\text { Consulto los correos electrónicos, el blog, el sitio Web o el medio utilizado } \\
\text { por el centro educativo para seguir las actividades que sugieren los docentes } \\
\text { para los estudiantes. }\end{array}$ \\
\hline & $\begin{array}{l}\text { Mezclo mis propias ideas con el plan de la escuela sobre lo que la o el menor a } \\
\text { mi cargo necesita aprender. }\end{array}$ \\
\hline & $\begin{array}{l}\text { Sigo en las redes sociales lo que otros padres hacen y lo utilizo como una } \\
\text { inspiración, como referencia o guía. }\end{array}$ \\
\hline & $\begin{array}{l}\text { Tomo ideas de familiares o amigos utilizando otros medios de comunica- } \\
\text { ción (por }\end{array}$ \\
\hline & ejemplo, teléfono, WhatsApp, etc.). \\
\hline & Busco ideas en Internet utilizando diferentes sitios web. \\
\hline \multirow{5}{*}{$\begin{array}{l}\text { Prácticas educati- } \\
\text { vas formales }\end{array}$} & Trato de replicar la forma en que me enseñaron cuando estaba en la escuela \\
\hline & $\begin{array}{l}\text { Enumero, preparo y desarrollo las actividades yo misma(o) antes de desa- } \\
\text { rrollarlas con la o el menor a mi cargo. }\end{array}$ \\
\hline & Establezco junto con la o el menor a mi cargo un horario para la educación en \\
\hline & casa. \\
\hline & $\begin{array}{l}\text { Desarrollo con la o el menor a mi cargo actividades de aprendizaje espon- } \\
\text { táneas no necesariamente relacionadas con la escuela como cocinar, hacer } \\
\text { carpintería, jugar videojuegos, hacer actividades físicas, etc. }\end{array}$ \\
\hline \multirow{15}{*}{$\begin{array}{l}\text { Prácticas educa- } \\
\text { tivas no formales }\end{array}$} & Comprar online \\
\hline & Comer \\
\hline & Cocinar \\
\hline & Aprender algo nuevo de internet \\
\hline & Hablar sobre cosas que son importantes para la o el menor \\
\hline & Jugar en el jardín \\
\hline & Leer un libro impreso \\
\hline & Leer un libro electrónico o un artículo \\
\hline & Cantar \\
\hline & Ver películas y discutirlas \\
\hline & Crear una obra de arte \\
\hline & Usar la tecnología para crear o editar contenido \\
\hline & Jugar videojuegos en el computador \\
\hline & Contactar a amigos o familiares mediante el uso de Internet \\
\hline & Interactuar con otros en las redes sociales \\
\hline
\end{tabular}

Elaboración propia a partir ICIPES, 2020. 


\subsection{Plan de análisis}

Para conocer las prácticas educativas que utiliza la clase media en Chile en comparación a otras clases económicas se realiza una división considerando los años de escolaridad alcanzados por los padres y madres del menor. En esta línea, debido al diseño no probabilístico de la encuesta y con el objetivo de disminuir el sesgo de muestreo existente por la subrepresentación de grupos sociales, se aplican ponderadores en la muestra según el nivel educativo de los y las participantes. Estos pesos se elaboran a partir de los datos disponibles de la Encuesta de Caracterización Socioeconómica Nacional (CASEN) para 2017.

Gráfico 1. Nivel educacional de madres y padres

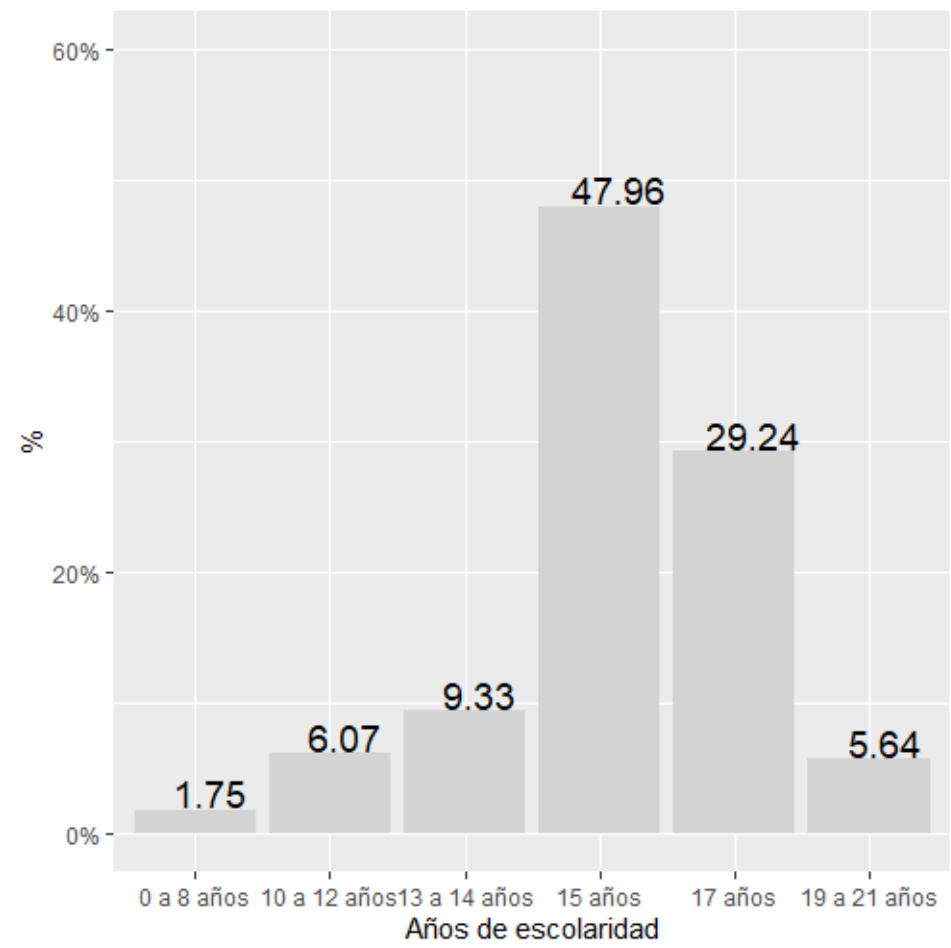

Fuente: Elaboración propia a partir ICIPES, 2020.

Con respecto a la división de clases sociales, se generan dos grupos. El primero agrupa a las personas sin estudios formales y aquellas que alcanzan 14 años de escolaridad, al cual se le denomina, grupo no profesional. En contraste, el segundo grupo se clasifica como profesional y engloba a los apoderados con 15 hasta 21 años de educación. Al revisar las características de ambos, se observa especiales diferencias en términos de ingreso (gráfico 1), más que en otro ámbito. 
Gráfico 2. Ingreso total del hogar en el último mes

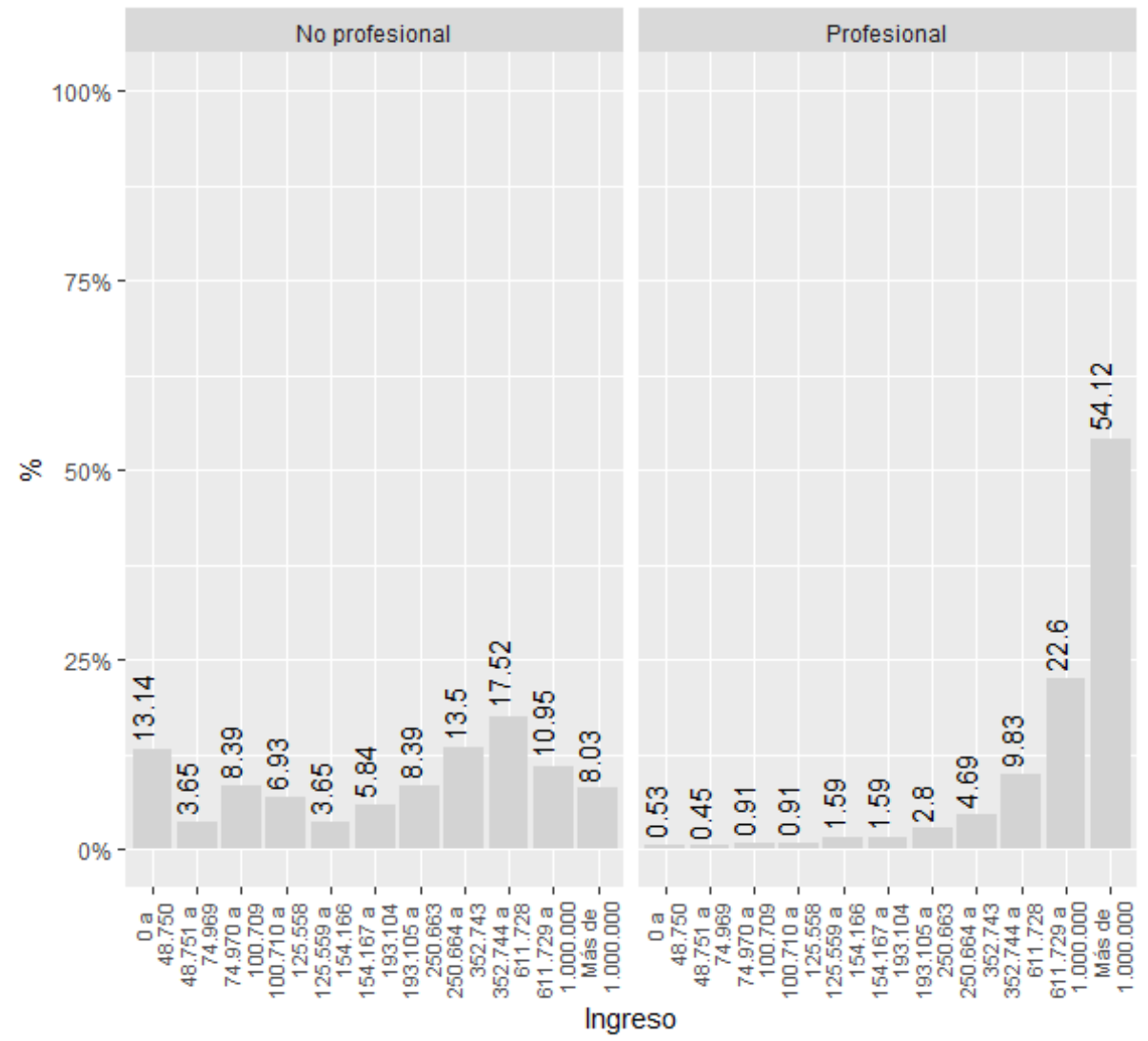

Fuente: Elaboración propia a partir ICIPES, 2020.

Al revisar otras características, tal como el género del apoderado según grupo, en ambos la mayoría son mujeres, $79 \%$ y $91 \%$, profesionales y no profesionales, respectivamente. En relación con la edad, los dos grupos se concentran en los 35 - 44 años (47\% grupo profesional y $43 \%$ no profesional) y 45 - 54 años (33\% profesionales y $29 \%$ no profesionales). La composición del grupo familiar es similar en los dos conjuntos, la mayoría vive con el padre o madre de su menor o bien, no tiene una pareja estable. Con respecto al menor, sus edades son variadas, pero en los dos grupos hay más adolescentes de 13 y 14 años. Por último, tanto en el conjunto de profesionales (44\%) y no profesionales (49\%) se envía diariamente el plan de aprendizaje o actividades por parte de la escuela. En definitiva, la división social realizada se justifica de acuerdo con los años de escolaridad alcanzado y sus diferencias de ingresos mensuales, más que por otras características.

De manera complementaria al análisis descriptivo se ejecuta un test de independencia, chi cuadrado. La independencia estadística refiere a cuando la distribución condicional de dos variables, en una población, son idénticas, o bien que la probabilidad 
de la muestra difiera de la probabilidad de la población (Agresti, 2018). En este estudio, buscamos ver si las prácticas educativas - o algunas de ellas - de las familias chilenas están relacionadas con su clase social $\left(\mathrm{H}_{\mathrm{a}}\right) \mathrm{O}$, al contrario, están disociadas $\left(\mathrm{H}_{0}\right)$.

\section{Resultados}

Para cumplir con las exigencias del proceso de aprendizaje remoto, las familias recurrieron a diversas prácticas que complementan los recursos entregados por la escuela. Entre estas prácticas de fortalecimiento educativo se encuentra i) consultar sitios oficiales que sugieren los docentes, ii) implementar ideas propias, iii) utilizar redes sociales para conocer la experiencia de otros apoderados y usarlo como guía, iv) tomar ideas familiares o de amigos o solamente v) buscar en internet. De este grupo de prácticas, las últimas tres están relacionadas con la clase social, observando diferencias en la práctica de tomar ideas de familiares o amigos a través de dispositivos tecnológicos. Para Chile, los resultados muestran que el $29 \%$ de los apoderados del grupo no profesional aplican esta acción, mientras que $14 \%$ de los profesionales lo harían. Cabe señalar que el 63\% de los padres de clase profesional rara vez consulta a familiares o amigos, lo que también podría relacionarse con su capital cultural. También es importante notar que el grupo de no profesionales (53\%) en comparación a los profesionales (41\%) sería más recurrente a buscar en internet ideas para fortalecer el proceso educativo del menor.

Tabla 2. Distribución de las prácticas de fortalecimiento educativo utilizadas por la familia en el hogar

Frecuentemente Ocasionalmente Rara vez $\begin{gathered}\text { Chi-cuadrado } \\ (\mathrm{p} \text {-value) }\end{gathered}$

Consulto los correos electrónicos, el blog, el sitio Web o el medio utilizado por el centro educativo para seguir las actividades que sugieren los docentes para los estudiantes.

\begin{tabular}{|l|c|c|c|c|}
\hline Profesional & $321(77,93 \%)$ & $61(14,97 \%)$ & $29(7,11 \%)$ & 0.288 \\
\hline No profesional & $877(74,09 \%)$ & $203(17,15 \%)$ & $103(8,76 \%)$ &
\end{tabular}

Mezclo mis propias ideas con el plan de la escuela sobre lo que la o el menor a mi cargo necesita aprender.

\begin{tabular}{|l|c|c|c|c|}
\hline Profesional & $183(44,60 \%)$ & $88(21,54 \%)$ & $139(33,86 \%)$ & 0.215 \\
\hline No profesional & $544(45,99 \%)$ & $289(24,45 \%)$ & $350(29,56 \%)$ & \\
\hline
\end{tabular}

Sigo en las redes sociales lo que otros padres hacen y lo utilizo como una inspiración, como referencia o guía.

\begin{tabular}{|l|r|r|r|r|}
\hline Profesional & $41(10,05 \%)$ & $73(17,76 \%)$ & $297(72,18 \%)$ & 0.000 \\
\hline No profesional & $211(17,88 \%)$ & $246(20,80 \%)$ & $726(61,31 \%)$ & \\
\hline
\end{tabular}

Tomo ideas de familiares o amigos utilizando otros medios de comunicación (por ejemplo, teléfono, WhatsApp, etc.).

\begin{tabular}{l|c|c|c|c}
\hline Profesional & $59(\mathbf{1 4 , 4 4 \% )}$ & $93(22,60 \%)$ & $259(62,96 \%)$ & 0.000 \\
\hline No profesional & $345(29,20 \%)$ & $315(26,64 \%)$ & $523(44,16 \%)$ & \\
\hline \begin{tabular}{l|l|l|l|} 
Busco ideas en Internet utilizando diferentes sitios web. \\
Profesional
\end{tabular} $167(40,67 \%)$ & $106(25,93 \%)$ & $137(33,41 \%)$ & 0.000 \\
\hline No profesional & $622(52,55 \%)$ & $307(25,91 \%)$ & $255(21,53 \%)$ & \\
\hline
\end{tabular}
Fuente: Elaboración propia a partir ICIPES, 2020 
Por otra parte, y respecto de las prácticas referidas a la estructuración del proceso educativo, se distinguen las siguientes opciones: i) replicar la forma en que le enseñaron ii) preparar el estudio y iii) desarrollar actividades de aprendizaje espontáneo con el menor. Como se puede observar en la tabla 2, estas tres acciones son realizadas frecuentemente por los apoderados del grupo no profesional y en menor medida por el grupo profesional. Hipotetizando, se podría considerar el factor de la dependencia administrativa de la escuela, debido a que las instituciones del sector privado presentan una mayor frecuencia de clases virtuales (Elige Educar, 2020a,b), lo que disminuiría la ejecución de labores extras por parte de padres y madres en aprendizaje remoto.

Tabla 3. Distribución de las prácticas educativas formales utilizadas por la familia en el hogar

\begin{tabular}{|c|c|c|c|c|}
\hline & Frecuentemente & Ocasionalmente & Rara vez & $\begin{array}{l}\text { Chi-cuadrado } \\
\text { (p-value) }\end{array}$ \\
\hline \multicolumn{5}{|c|}{ Trato de replicar la forma en que me enseñaron cuando estaba en la escuela. } \\
\hline Profesional & $90(21,84 \%)$ & $141(34,24 \%)$ & $180(43,92 \%)$ & \multirow[t]{2}{*}{0.000} \\
\hline No profesional & $501(42,34 \%)$ & $350(29,56 \%)$ & $333(28,10 \%)$ & \\
\hline \multicolumn{5}{|c|}{$\begin{array}{l}\text { Enumero, preparo y desarrollo las actividades yo misma(o) antes de desarrollarlas con la o el } \\
\text { menor a mi cargo. }\end{array}$} \\
\hline Profesional & $115(28,04 \%)$ & $89(21,77 \%)$ & $206(50,19 \%)$ & \multirow[t]{2}{*}{0.003} \\
\hline No profesional & $423(35,77 \%)$ & $272(22,99 \%)$ & $488(41,24 \%)$ & \\
\hline \multicolumn{5}{|c|}{ Establezco junto con la o el menor a mi cargo un horario para la educación en casa. } \\
\hline Profesional & $282(68,56 \%)$ & $72(17,69 \%)$ & $56(13,76 \%)$ & \multirow[t]{2}{*}{0.325} \\
\hline No profesional & $774(65,33 \%)$ & $211(17,88 \%)$ & $198(16,79 \%)$ & \\
\hline \multicolumn{5}{|c|}{$\begin{array}{l}\text { Desarrollo con la o el menor a mi cargo actividades de aprendizaje espontáneas no necesaria } \\
\text { mente relacionadas con la escuela como cocinar, hacer carpintería, jugar videojuegos, hace } \\
\text { actividades físicas, etc. }\end{array}$} \\
\hline Profesional & $275(66,82 \%)$ & $92(22,45 \%)$ & $44(10,73 \%)$ & \multirow[t]{2}{*}{0.003} \\
\hline No profesional & $890(75,18 \%)$ & $190(16,06 \%)$ & $103(8,76 \%)$ & \\
\hline
\end{tabular}

Fuente: elaboración propia a partir ICIPES, 2020.

Por último, consideramos relevante indagar en las actividades extra-educativas a las que están expuestos los menores al estar en el hogar y que las familias potencian. En general, los resultados muestran que los padres y madres profesionales son más asiduos a promover este tipo de prácticas en comparación a los no profesionales. Tal hallazgo se condice con la literatura y estudios recientes, que vincula la realización de prácticas informales de educación o extracurriculares con las lógicas o recursos de las familias de clases medias profesionales (Lareau, 2011; Bonal y González, 2020). Cabe mencionar que, del listado de prácticas no formales, las que no presentan diferencias según clase social son las de cantar y jugar videojuegos, mientras que las actividades que se observaron mayores diferencias se encuentran comprar en línea, aprender sobre algo en internet, leer un libro impreso o leer un libro electrónico, lo que también podría relacionarse con la relevancia que le otorga a la escuela a ciertas prácticas sobre otras. 
Tabla 4. Distribución de las prácticas educativas no formales utilizadas por la familia en el hogar

\begin{tabular}{c|c|c} 
Sí & No & $\begin{array}{c}\text { Chi-cuadrado } \\
(p-v a l u e)\end{array}$ \\
\hline
\end{tabular}

\section{Comprar online}

Profesional

$231(56,24 \%)$

$180(43,76 \%)$

0.000

No profesional

$380(32,12 \%)$

$804(67,88 \%)$

Comer

\begin{tabular}{l|l} 
Profesional \\
No profesional \\
Cocinar
\end{tabular}

\begin{tabular}{|l|l|l|l|}
\hline Profesional & $369(89,80 \%)$ & $42(10,20 \%)$ & 0.000 \\
\hline No profesional & $960(81,02 \%)$ & $224(18,98 \%)$ & \\
\hline
\end{tabular}

Hablar sobre cosas que son importantes para la o el menor

\begin{tabular}{|c|c|c|c|}
\hline Profesional & $386(93,80 \%)$ & $25(6,20 \%)$ & \multirow[t]{2}{*}{0.000} \\
\hline No profesional & $1020(86,13 \%)$ & $164(13,87 \%)$ & \\
\hline \multicolumn{4}{|c|}{ Aprender sobre algo en Internet } \\
\hline Profesional & $290(70,52 \%)$ & $121(29,48 \%)$ & \multirow[t]{2}{*}{0.000} \\
\hline No profesional & $678(57,30 \%)$ & $505(42,70 \%)$ & \\
\hline \multicolumn{4}{|c|}{ Jugar en el jardín } \\
\hline Profesional & $208(50,49 \%)$ & $203(49,51 \%)$ & \multirow[t]{2}{*}{0.000} \\
\hline No profesional & $410(34,67 \%)$ & $774(65,33 \%)$ & \\
\hline \multicolumn{4}{|c|}{ Leer un libro impreso } \\
\hline Profesional & $270(65,61 \%)$ & $141(34,39 \%)$ & \multirow[t]{2}{*}{0.000} \\
\hline No profesional & $441(37,23 \%)$ & $743(62,77 \%)$ & \\
\hline \multicolumn{4}{|c|}{ Leer un libro electrónico o un artículo } \\
\hline Profesional & $168(40,89 \%)$ & $243(59,11 \%)$ & \multirow[t]{2}{*}{0.000} \\
\hline No profesional & $302(25,55 \%)$ & $882(74,45 \%)$ & \\
\hline \multicolumn{4}{|c|}{ Ver una película y discutirla } \\
\hline Profesional & $370(89,87 \%)$ & $41(10,13 \%)$ & \multirow[t]{2}{*}{0.021} \\
\hline No profesional & $1011(85,40 \%)$ & $172(14,60 \%)$ & \\
\hline \multicolumn{4}{|l|}{ Cantar } \\
\hline Profesional & $239(58,05 \%)$ & $172(41,95 \%)$ & \multirow[t]{2}{*}{0.364} \\
\hline No profesional & 657 (55,47\%) & 527 (44,53\%) & \\
\hline
\end{tabular}

Crear una obra de arte en papel o utilizando cualquier otro material físico

\begin{tabular}{|l|l|l|l|}
\hline Profesional & $183(44,44 \%)$ & $228(55,56 \%)$ & 0.000 \\
\hline No profesional & $389(32,85 \%)$ & $795(67,15 \%)$ & \\
\hline
\end{tabular}

Usar la tecnología para crear o editar videos, fotos o música u otro contenido

\begin{tabular}{l|l|l|l|}
\hline Profesional & $218(53,06 \%)$ & $193(46,94 \%)$ & 0.000 \\
\hline No profesional & $514(43,43 \%)$ & $670(56,57 \%)$ & \\
\hline
\end{tabular}




\begin{tabular}{|c|c|c|c|}
\hline & Sí & No & $\begin{array}{l}\text { Chi-cuadrado } \\
\text { (p-value) }\end{array}$ \\
\hline \multicolumn{4}{|c|}{$\begin{array}{l}\text { Contactar a amigos o familiares mediante el uso de Internet (por ejemplo, Skype, } \\
\text { FaceTime, WhatsApp, etc,) }\end{array}$} \\
\hline Profesional & $366(89,04 \%)$ & $45(10,96 \%)$ & \multirow[t]{2}{*}{0.000} \\
\hline No profesional & $951(80,29 \%)$ & $233(19,71 \%)$ & \\
\hline \multicolumn{4}{|c|}{ Jugar videojuegos en la computadora } \\
\hline Profesional & $187(45,58 \%)$ & $224(54,42 \%)$ & \multirow[t]{2}{*}{0.530} \\
\hline No profesional & $518(43,80 \%)$ & $666(56,20 \%)$ & \\
\hline \multicolumn{4}{|c|}{$\begin{array}{l}\text { Interactuar en las redes sociales (por ejemplo, comentar las publicaciones de los } \\
\text { demás) }\end{array}$} \\
\hline Profesional & $166(40,44 \%)$ & $245(59,56 \%)$ & \multirow[t]{2}{*}{0.000} \\
\hline No profesional & 363 (30,66\%) & $821(69,34 \%)$ & \\
\hline
\end{tabular}

Fuente: elaboración propia a partir ICIPES, 2020

A modo de síntesis, los resultados muestran que existe un involucramiento parental transversal en el proceso y experiencia de aprendizaje en el contexto de educación remota. Sin embargo, existen diferencias en las prácticas educativas para enfrentar la escolaridad en el hogar. Así, mientras que los padres y madres no profesionales buscan prácticas de fortalecimiento educativo, aquellos que componen el grupo profesional priorizan la participación del menor en actividades extra-educativas que estimulen su aprendizaje cognitivo. Finalmente, se encuentra que ciertas prácticas educativas formales y no formales están asociadas con la clase social de las familias y, por ende, se reafirma la desigualdad educativa que se reproduce según los recursos económicos que tiene una persona.

\section{Discusión y conclusión}

El involucramiento parental en la educación de las niñas, niños y adolescentes se ha vuelto un factor clave en el curso de la presente pandemia. Las madres y los padres cuidadores se han transformado en mediadores del aprendizaje escolar, ya sea a través del acompañamiento y monitoreo de actividades escolares virtuales, o bien mediante otros medios en caso de no contar con Internet.

A pesar de la preocupación generalizada y transversal de las familias por el aprendizaje de sus hijos o hijas, se presentan importantes diferencias de apoyo parental entre las clases sociales de profesionales y la de no profesionales. Estas diferencias se aprecian particularmente en relación con atributos del capital cultural y económico. Así, los padres y madres de familia no profesionales tienden a buscar más apoyo entre familiares y amigos profesionales para las tareas escolares, tienen el doble de probabilidades de replicar la forma de enseñanza que experimentaron en la escuela, tienen menor acceso a internet, así como a libros y a obras artísticas que puedan servir de actividad extra-curricular para los estudiantes.

Las tendencias vistas en este artículo muestran un doble fenómeno. En primer lugar, muestran que las madres y padres, independientemente de su nivel de estudios, están interesados en apoyar la continuidad educativa de sus hijos en pandemia. En segundo lugar, se aprecian desigualdades que favorecen a los padres y madres de la 
clase profesional, quienes tienen más acceso a recursos y conocimientos culturales y académicos que les permiten apoyar de mejor forma la educación de sus hijos e hijas. En este sentido, vale la pena preguntarse por el valor de la educación en la vida adulta, no solo en términos de la contribución económica y social que esta puede hacer, sino también respecto de cómo más años de escolarización representan una forma de acumular y heredar mejores apoyos y habilidades para las hijas e hijos.

Con el objetivo de explorar las prácticas educativas de la clase media-profesional chilena en período de crisis sanitaria, este artículo presenta diversas experiencias y acciones que realizan las familias en sus hogares a partir de su nivel de escolaridad. Reafirmando literatura producida hace varias décadas, este artículo muestra que las desigualdades económicas en el sistema educativo se reproducen e incluso aumentan en contextos de aprendizaje remoto, o bien, cuando la escuela, como institución socializadora, pierde protagonismo. Ahora bien, este estudio presenta ciertas limitaciones que son importantes de visualizar para ser subsanadas en investigaciones futuras. En primer lugar, la edad de los menores es amplia, existiendo alta proporción de adolescentes, que quizás no experimenten - por su etapa escolar - las prácticas educativas mencionadas. En este sentido, es importante focalizarse en la educación inicial o rangos etarios menores, ya que son quienes más necesitan del apoyo parental en contextos de emergencia. Una segunda limitación refiere al nivel de representatividad del estudio, si bien, se aplican factores de expansión para mejorar su alcance, es necesario generar estudios con una muestra probabilísticas y alta tasa de respuesta. Trabajar en nuevas investigaciones, que permitan subsanar estas limitaciones permitirá, en un futuro próximo, continuar aportando al conocimiento actual sobre el sistema educacional y el rol de la familia, así como al entendimiento de las brechas educativas de Chile y del continente.

\section{Agradecimientos}

Los y las autoras agradecen el apoyo del proyecto PIA CIE 160007 de la Agencia Nacional de Investigación y Desarrollo de Chile

\section{Referencias}

Agresti, A. (2018). Statistical Methods for the Social Sciences. (5 $5^{\text {th }}$ edition). United States: University of Florida.

Bellei, C. (2015). El gran experimento. Mercado y privatización de la educación chilena. Santiago, Chile: LOM.

Bhamani, S., Makhdoom, A. Z., Bharuchi, V., Ali, N., Kaleem, S. y Ahmed, D. (2020). Home learning in times of COVID: Experiences of parents. Journal of Education and Educational Development, 7(1), 9-26. https://doi.org.10.22555/joeed.v7i1.3260

Bonal, X. y González, S. (2020). The impact of lockdown on the learning gap: Family and school divisions inn times of crisis. International Review of Education, 66, 635-655. https://doi. org/10.1007/s11159-020-09860-z

Bubb, S. y Jones, M.A. (2020). Learning from the COVID-19 home-schooling experience: Listening to pupils, parents/carers and teachers. Improving Schools, 23(3), 209-222. https://doi. org/10.1177/1365480220958797 
Bourdieu, P. y Passeron, J.C. (1964). Les heritiers. Paris: Les Editions de Minuit.

Busso, M. y Messina, J. (2020). La crisis de la desigualdad. América Latina y el Caribe en la encrucijada. Banco Interamericano del Desarrollo. Recuperado de https://bit.ly/3fl9tjy

Centro de Investigación Avanzada en Educación, Centro de Investigación para la Educación Inclusiva y EduGlobal (2020). Covid-19. Nuevos contextos, nuevas demandas y experiencia docente en Chile. Recuperado de https://bit.ly/3hRZZOx

CEPAL y UNESCO. (2020). La educación en tiempos de la pandemia de Covid-19. Recuperado de: https://bit.ly/2SuiOHB

Educación 2020. (2020). Informe de resultados Estamos Conectados. Testimonios y experiencias de las comunidades educativa ante la crisis sanitaria. Recuperado de https://bit.ly/3wrZhes

Elige Educar (2020a). Situación de docentes y educadores en contexto de pandemia. Reporte de resultados 19 de mayo 2020. Recuperado de https://bit.ly/2SuiOHB

Elige Educar (2020b). Situación de docentes y educadores en contexto de pandemia. Versión 2, septiembre 2020. Recuperado de https://bit.ly/3vmGC3R

Fontenelle-Tereshchuk, D. (2021). 'Homeschooling'and the COVID-19 Crisis: The Insights of Parents on Curriculum and Remote Learning. Interchange, 1-25. https://doi.org/10.1007/s10780021-09420-w

Gelber, D., Castillo, C., Alarcón, L., Treviño, E. y Escribano, R. (2021). Covid-19 and the right to education in Chile: An opportunity to revisit our social contrast. International Review of Education. https://doi.org/10.1007/s11159-021-09881-2

Goodall, J. y Montgomery, C. (2014). Parental involvement to parental engagement: A continuum. Educational review, 66(4), 399-410. https://doi.org/10.1080/00131911.201 $\underline{3.781576}$

Gubbins, V. y Otero, G. (2020). Parental involvement and low-SES children's academic achievement in early elementary school: new evidence from Chile. Educational Studies, 46(5), 548-569. https://doi.org/10.1080/03055698.2019.1620691

Lareau, A. (2011). Unequal childhoods: Class, race and family life. California: University California Press.

Lareau, A. (2000). Home advantage. Social class and parental intervention in elementary education. Maryland: Rowman \& Littlefield Publishers.

Ministerio de Educación Chile (2021). Primer informe Consejo Asesor Paso a Paso abramos las escuelas. Recuperado de https://bit.ly/34hO9Fg

Ministerio de Educación Chile (2020). Resumen estadístico de la educación 2019. Recuperado de https://bit.ly/3wzHQsO

Nogueira, M.A. (2010). A revisited theme: Middle classes and the school. En M. Apple, S.J. Ball y L.A. Gandin (eds.), The routledge international handbook of the sociology of education (pp. 253-63). Abingdon: Routledge.

OECD (2019a). Education at a Glance 2019: OECD Indicators. Paris: OECD Publishing.

OECD (2019b). Balancing school choice and equity: An international perspective based on Pisa. París: OECD Publishing.

OECD (2020). Making the most of technology for learning and training in Latin America. Paris: OECD.

Osorio-Saez, E., Eryilmaz, N., Sandoval-Hernandez, A., Lau, Y.Y., Barahona, E., Anwar-Bhatti,A., ... Zionts, A. (2021). Survey data on the impact of COVID-19 on parental engagement across 23 countries. Data in Brief 35(1), 1 - 18, https://doi.org/10.1016/j.dib.2021.106813

Parcerisa, L. y Falabella, A. (2017). La consolidación del estado evaluador a través de políticas de rendición de cuentas: Trayectoria, producción y tensiones en el sistema educativo chileno. Archivos Analíticos de Políticas Educativas, 24(89), 1-24. https://doi.org/10.14507/ epaa.25.3177

Parczewska, T. (2020). Difficult situations and ways of coping with them in the experiences of parents homeschooling their children during the COVID-19 pandemic in Poland. Education 3-13, 1-12. https://doi.org/10.1080/03004279.2020.1812689 
Ponce, T., Bellei, C. y Vielma, C. (2020). Experiencias educativas en casa de niñas y niños durante la pandemia Covid-19. Recuperado de https://bit.ly/3bTe3Do

Pozas, M., Letzel, V. y Schneider, C. (2021). 'Homeschooling in times of corona': exploring Mexican and German primary school students' and parents' chances and challenges during homeschooling. European Journal of Special Needs Education, 1-16. https://doi.org/10. 1080/08856257.2021.1874152

Reay, D., Crozier, G. y James, D. (2011). White middle-class identities and urban schooling. Londres: Palgrave Macmillan.

Santiago, P., Fiszbein, A., García, S y Radinger, T. (2017). OECD reviews of school resources: Chile 2017. Paris: OECD Publishing.

Skwarchuk, S. L., Sowinski, C. y LeFevre, J. A. (2014). Formal and informal home learning activities in relation to children's early numeracy and literacy skills: The development of a home numeracy model. Journal of experimental child psychology, 121, 63-84. https://doi. org/10.1016/j.jecp.2013.11.006

Treviño, E. (2018). Diagnóstico del Sistema Escolar: Las reformas educativas 2014-2017. En Sánchez, I. (ed.). Ideas en educación II. Definiciones en tiempos de cambio. Santiago de Chile: Ediciones Universidad Católica de Chile (pp. 135-165).

UNESCO. (2020). Global Education Monitoring Report 2020: Inclusion and education, all means all. París: Unesco.

UNESCO. (2021). Unesco figures show two thirds of an academic year lost on average worldwide due to Covid-19 school closures. Recuperado de https://bit.ly/3oPXvkl

Villalobos, C. y Quaresma, M.L. (2015). Sistema escolar chileno. Características y consecuencias de un modelo orientado al mercado. Convergencia. Revista de Ciencias Sociales, 69(3), 63-84.

Vincent, C. (2001). Social class and parental agency. Journal of Education Policy, 16(4), 347-364. https://doi.org/10.1080/0268093011-54344

Wilder, S. (2014). Effects of parental involvementonacademic achievement: a meta-synthesis. Educational Review, 66(3), 377-397. https://doi.org/10.1080/00131911.2013.780009 